\title{
Some inverse scattering problems for perturbations of the biharmonic operator
}

\author{
Valery Serov \\ University of Oulu, Finland
}

October 11, 2019

\begin{abstract}
Some inverse scattering problems for the three-dimensional biharmonic operator are considered. The operator is perturbed by first and zero order perturbations, which may be complex-valued and singular. We show the existence of the scattering solutions in the Sobolev space $W_{\infty}^{1}\left(R^{3}\right)$. One of the main result of this paper is the proof of analogue of Saito's formula (in different form as known before), which can be used to prove a uniqueness theorem for the inverse scattering problem. Another main result is to obtain the estimates for the kernel of the resolvent of the direct operator in $W_{\infty}^{1}$ and to prove the reconstruction formula for the unknown coefficients of this perturbation.
\end{abstract}

\section{Introduction}

We consider the following three-dimensional biharmonic operator

$$
H_{4} u(x)=\Delta^{2} u(x)+\vec{W}(x) \cdot \nabla u(x)+V(x) u(x)=0,
$$

where $\Delta$ is the Laplacian and - denotes the dot-product in $R^{3}$ for complexvalued vectors in $C^{3}$. The bi-Laplacian is perturbed by the first and zero order perturbations, vector-valued function $\vec{W}$ and a scalar function $V$, that may be complex-valued and very singular. More precisely, we assume that $\vec{W}$ belongs to $L^{\infty}\left(R^{3}\right)$ and $V$ belongs to the Kato space $K_{3}$, i.e.

$$
\sup _{x \in R^{3}} \int_{|x-y| \leq 1} \frac{|V(y)|}{|x-y|} d y<\infty,
$$

and both have special behaviour at the infinity

$$
|\vec{W}(x)|, \quad|V(x)| \leq \frac{C}{|x|^{\mu}}, \quad|x| \geq R, \quad \mu>3,
$$

where $C>0$, and $R>0$ is big enough. 
The motivation to study operators of order four appears for example in the study of elasticity and the theory of vibration of beams. As a concrete example, the linear beam equation [2]

$$
\partial_{t}^{2} U(x, t)+\Delta^{2} U(x, t)+m(x) U(x, t)=0,
$$

under time-harmonic assumptions $U(x, t)=u(x) e^{-i \omega t}$ leads to the equation

$$
\Delta^{2} u(x)+m(x) u(x)=\omega^{2} u(x) .
$$

The wave parameter $\omega$ is fixed (in general) here, nevertheless we can consider it fixed but big enough. This allows to consider some scattering problems with high frequency for this potential equation. In particular, we can use some numerical methods in that case. For the scattering problems (including linear or nonlinear equations), see for example [8] and references therein. In terms of inverse problems for bi- and poly-harmonic operators it might be mentioned some solutions to inverse boundary value problems, see for example [6]. One can refer also to [13], where the fundamental result concerning the global uniqueness for an inverse boundary value problem was proved. For the operators with vector potential one can mention [14].

The present work is concerned with the following scattering problem for operator $\mathrm{H}_{4}$ given by

$$
H_{4} u(x)=k^{4} u(x), \quad u(x)=u_{0}(x)+u_{s c}(x), \quad u_{0}(x)=e^{i k x \cdot \theta}, \quad \theta \in S^{2},
$$

where scattered wave $u_{s c}$ and its Laplacian $\Delta u_{s c}$ are required to satisfy Sommerfeld radiation condition at the infinity

$$
\lim _{r \rightarrow \infty} r^{\frac{n-1}{2}}\left(\frac{\partial f(x)}{\partial r}-i k f(x)\right)=0, \quad r=|x|, \quad f=u_{s c} \quad \text { or } \quad f=\Delta u_{s c} .
$$

The author was originally motivated to start studying scattering for fourth order operators by the article [1] (see also [15]), where the time-evolution of several scattering coefficients for the one-dimensional biharmonic operator were studied. In terms of inverse scattering problems for fourth order operator might be mentioned Iwasaki's results [4], [5]. In these works Iwasaki studied the scattering problem in one-dimensional case and considered the inverse problem as a Riemann-Hilbert boundary value problem with respect to the wave number $k$ in the complex cone $\arg \left(\left[0, \frac{\pi}{4}\right]\right) \backslash\{0\}$.

The main differences of present work is that all considered scattering problems are studying here in the usual Sobolev spaces (compare with the weighted Sobolev spaces in the previous publications) and that the possible local singularities of the unknown coefficients $\vec{W}$ and $V$ are stronger than it was considered before. Another important difference is concerned to Theorem 2, where the inverse scattering problem in terms of the Green's functions is considered.

We are looking for the scattering solutions $u_{s c}$ to the equation (4) in the Sobolev space $W_{\infty}^{1}\left(R^{3}\right)$. Under the Sommerfeld radiation conditions (5) the 
scattering solutions to equation (4) are the unique solutions of the integral Lippmann-Schwinger equation (see [15] for details)

$$
u(x)=u_{0}(x)-\int_{R^{3}} G_{k}^{+}(|x-y|)(\vec{W}(y) \cdot \nabla u(y)+V(y) u(y)) d y,
$$

where $G_{k}^{+}$is the outgoing fundamental solution of the operator $\left(\Delta^{2}-k^{4}\right)$ in $R^{3}$, i.e., the kernel of the integral operator $\left(\Delta^{2}-k^{4}-i 0\right)^{-1}$. This function $G_{k}^{+}$in $R^{3}$ has the following form

$$
G_{k}^{+}(|x|)=\frac{e^{i k|x|}-e^{-k|x|}}{8 \pi k^{2}|x|}, \quad k>0 .
$$

Since $u_{0}$ is just a bounded function with the norm $\left\|u_{0}\right\|_{L^{\infty}\left(R^{3}\right)}=1$ it is more convenient to study (in stead of (6)) the equivalent integral equation for the scattered wave, namely

$u_{s c}(x)=\tilde{u}_{0}(x)-\int_{R^{3}} G_{k}^{+}(|x-y|)\left(\vec{W}(y) \cdot \nabla u_{s c}(y)+V(y) u_{s c}(y)\right) d y=: \tilde{u}_{0}+L_{k}\left(u_{s c}\right)$,

where $\tilde{u}_{0}=L_{k}\left(u_{0}\right)$. As it is shown (see [15]) that a solution to the scattering problem (4), (5) also satisfies equation (6). This translates the study of the scattering problem to the study of integral equation $(6)((8))$. It will be shown that this solution admits for fixed $k>0$ asymptotic representation

$$
u(x, k, \theta)=e^{i k x \cdot \theta}+C \frac{e^{i k|x|}}{k^{2}|x|} A\left(k, \theta^{\prime}, \theta\right)+o\left(\frac{1}{|x|}\right), \quad|x| \rightarrow \infty,
$$

where $\theta, \theta^{\prime}=\frac{x}{|x|} \in S^{2}, C$ is known constant, and function $A\left(k, \theta^{\prime}, \theta\right)$ is called a scattering amplitude and defined by

$$
A\left(k, \theta^{\prime}, \theta\right):=\int_{R^{3}} e^{-i k \theta^{\prime} \cdot y}(\vec{W}(y) \cdot \nabla u(y, k, \theta)+V(y) u(y, k, \theta)) d y .
$$

From the point of view of inverse problems one regards this scattering amplitude as one possible scattering data. For these purposes one requires the scattering amplitude to be known for all possible angles $\theta$ and $\theta^{\prime}$ and all arbitrarily high frequencies ( $k>0$ large). Then Saito's formula is given by the following theorem.

Theorem 1 (Saito's formula) Assume that $\vec{W}$ belongs to $L^{\infty}\left(R^{3}\right), V$ belongs to the Kato space $K_{3}$ and both satisfy conditions (3). Then the limit

$$
\lim _{k \rightarrow+\infty} k^{2} \int_{S^{2} \times S^{2}} e^{-i k\left(\theta-\theta^{\prime}\right) \cdot x} A\left(k, \theta^{\prime}, \theta\right) d \theta d \theta^{\prime}=
$$




$$
=4 \pi^{2} \nabla_{x} \int_{R^{3}} \frac{\vec{W}(y)}{|x-y|^{2}} d y+8 \pi^{2} \int_{R^{3}} \frac{V(y)}{|x-y|^{2}} d y
$$

in the sense of distributions.

The significance of Saito's formula for inverse problems is apparent from its corollary.

Corollary 1 (Uniqueness) Let $\vec{W}_{1}, V_{1}$ and $\vec{W}_{2}, V_{2}$ be as in Theorem 1. Let, in addition, Fourier transform of $\vec{W}_{1}$ and $\vec{W}_{2}$ behaves as o $\left(|\xi|^{-1}\right)$ at the infinity. If the corresponding scattering amplitudes for these coefficients coincide for some sequence $k_{j} \rightarrow+\infty$ then

$$
V_{1}(x)-\frac{1}{2} \nabla \delta \star \vec{W}_{1}(x)=V_{2}(x)-\frac{1}{2} \nabla \delta \star \vec{W}_{2}(x)
$$

in the sense of tempered distributions, where $\delta$ is Dirac delta function and $\star$ denotes the convolution.

The limiting absorption principle can be applied for the operator $H_{4}$ to obtain the existence of the integral operator (see [15])

$$
\hat{G}_{p}:=\lim _{\epsilon \rightarrow+0}\left(H_{4}-k^{4}-i \epsilon\right)^{-1}
$$

such that the kernel $G_{p}(x, y, k)$ for $k>0$ large enough is the unique solution of the integral equation

$G_{p}(x, y, k)=G_{k}^{+}(x, y, k)-\int_{R^{3}} G_{k}^{+}(|x-z|)\left(\vec{W}(z) \cdot \nabla G_{p}(z, y, k)+V(z) G_{p}(y, z, k)\right) d z$,

From the point of view of inverse problems this kernel $G_{p}$ can be considered as another possible scattering data. More precisely the following theorem holds.

Theorem 2 (Reconstruction) Assume that $\vec{W}$ belongs to $L^{\infty}\left(R^{3}\right), V$ belongs to the Kato space $K_{3}$ and $V$ satisfies conditions (3) and, in addition, $\vec{W}$ satisfies this condition with $\mu>4$. Then for each fixed $\xi \in R^{3}$

$$
\begin{gathered}
\mathcal{F}^{-1}(V)(\xi)-\frac{i \xi}{2} \cdot \mathcal{F}^{-1}(\vec{W})(\xi)= \\
=\lim _{k,|x|,|y| \rightarrow+\infty} 32 \sqrt{2 \pi} k^{4}|x||y| e^{-i k(|x|+|y|)}\left(G_{k}^{+}(|x-y|)-G_{p}(x, y, k)\right)
\end{gathered}
$$

such that $\xi=k\left(\frac{x}{|x|}+\frac{y}{|y|}\right)$.

Corollary 2 (Uniqueness-II) Let $\vec{W}_{1}, V_{1}$ and $\vec{W}_{2}, V_{2}$ be as in Theorem 2. If the corresponding kernels $G_{p}^{(1)}(x, y, k)$ and $G_{p}^{(2)}(x, y, k)$ for these coefficients coincide for all $x, y$ large enough and some sequence $k_{j} \rightarrow+\infty$ then

$$
V_{1}(x)-\frac{1}{2} \nabla \delta \star \vec{W}_{1}(x)=V_{2}(x)-\frac{1}{2} \nabla \delta \star \vec{W}_{2}(x)
$$


in the sense of tempered distributions, where $\delta$ is Dirac delta function and * denotes the convolution.

This paper is organised as follows. In Section 2 some notations and estimates for $G_{k}^{+}$are recalled. Then it will be proved the existence and the uniqueness of the solutions to (6) and (11) together with asymptotic behaviour of the scattering solution $u$ and the kernel $G_{p}$. Several estimates for $u$ and $G_{p}$ are also given. Finally in Section 3 it will be given the proof of Theorem 1 and 2 .

\section{Solvability of direct scattering problems}

We use the usual definitions of the Sobolev space $W_{\infty}^{1}$ and the Lebesgue spaces $L^{p}, 1 \leq p \leq \infty$. We use also the following definitions for three-dimensional Fourier transform $\mathcal{F}$ and inverse Fourier transform $\mathcal{F}^{-1}$ :

$$
\mathcal{F}(f)(\xi)=\frac{1}{\sqrt{(2 \pi)^{3}}} \int_{R^{3}} f(x) e^{i x \cdot \xi} d x, \quad \mathcal{F}^{-1}(f)(x)=\frac{1}{\sqrt{(2 \pi)^{3}}} \int_{R^{3}} f(x) e^{-i x \cdot \xi} d \xi .
$$

Next, taking into account the definition $(7)$ of $G_{k}^{+}(|x|)$ in $R^{3}$ we obtain

$$
\left|G_{k}^{+}(|x|)\right| \leq \frac{1}{4 \pi k^{2}|x|}, \quad\left|\nabla G_{k}^{+}(|x|)\right| \leq \frac{1}{2 \pi k|x|}, \quad k>0, \quad x \in R^{3} .
$$

We now proceed to prove some estimates for the operator $L_{k}$.

Proposition 1 Let $\vec{W}$ belongs $L^{\infty}\left(R^{3}\right), V$ belongs to the Kato space $K_{3}$ and both satisfy conditions (3). Then the following properties are satisfied.

1. The function $\tilde{u}_{0}$ belongs to $W_{\infty}^{1}\left(R^{3}\right)$ with the estimates

$$
\left\|\tilde{u}_{0}\right\|_{L^{\infty}\left(R^{3}\right)} \leq \frac{c_{0}}{k}, \quad\left\|\nabla \tilde{u}_{0}\right\|_{L^{\infty}\left(R^{3}\right)} \leq c_{0}, \quad k \geq 1
$$

where constant $c_{0}$ is equal to

$$
\begin{gathered}
c_{0}=\frac{1}{2 \pi}\left(\|\vec{W}\|_{L^{\infty}\left(R^{3}\right)} C_{R}+C_{V}+2 C C_{\mu}\right), \quad C_{R}:=\sup _{x \in R^{3}} \int_{|y| \leq R} \frac{1}{|x-y|} d y, \\
C_{V}:=\sup _{x \in R^{3}} \int_{|y| \leq R} \frac{|V(y)|}{|x-y|} d y, \quad C_{\mu}:=\sup _{x \in R^{3}} \int_{|y| \geq R} \frac{1}{|x-y||y|^{\mu}} d y
\end{gathered}
$$

with $C, R, \mu$ are as in (3).

2. The operator $L_{k}: W_{\infty}^{1}\left(R^{3}\right) \rightarrow W_{\infty}^{1}\left(R^{3}\right)$ is bounded and satisfies for $k \geq 1$ the norm estimates

$$
\left\|L_{k} f\right\|_{L^{\infty}\left(R^{3}\right)} \leq \frac{c_{0}}{k^{2}}\|f\|_{W_{\infty}^{1}\left(R^{3}\right)}, \quad\left\|\nabla L_{k} f\right\|_{L^{\infty}\left(R^{3}\right)} \leq \frac{c_{0}}{k}\|f\|_{W_{\infty}^{1}\left(R^{3}\right)} .
$$


Proof Applying (13) one can obtain (see (14))

$$
\begin{aligned}
\left|\tilde{u}_{0}(x)\right| \leq & \frac{1}{4 \pi k^{2}}\left(\int_{|y| \leq R} \frac{k|\vec{W}(y)|+|V(y)|}{|x-y|} d y+C \int_{|y| \geq R} \frac{k+1}{|x-y||y|^{\mu}} d y\right) \leq \\
& \leq \frac{1}{4 \pi k}\left(\|\vec{W}\|_{L^{\infty}} C_{R}+C_{V}+2 C C_{\mu}\right) \leq \frac{c_{0}}{k}, \quad k \geq 1 .
\end{aligned}
$$

By similar method it can be proved that

$$
\left\|\nabla \tilde{u}_{0}\right\|_{L^{\infty}\left(R^{3}\right)} \leq c_{0}, \quad k \geq 1 .
$$

This proves 1 . Suppose now that $f \in W_{\infty}^{1}\left(R^{3}\right)$. Then

$$
\begin{aligned}
& \left|L_{k} f(x)\right| \leq \frac{1}{4 \pi k^{2}}\left(\int_{|y| \leq R} \frac{|\nabla f(y)||\vec{W}(y)|+|V(y)||f(y)|}{|x-y|} d y+C C_{\mu}\|f\|_{W_{\infty}^{1}}\right) \leq \\
& \leq \frac{1}{4 \pi k^{2}}\left(\|\nabla f\|_{L^{\infty}} \int_{|y| \leq R} \frac{\mid \vec{W}(y)}{|x-y|} d y+\|f\|_{L^{\infty}} \int_{|y| \leq R} \frac{|V(y)|}{|x-y|} d y+C C_{\mu}\|f\|_{W_{\infty}^{1}}\right) \leq \\
& \leq \frac{1}{4 \pi k^{2}}\left(\|\nabla f\|_{L^{\infty}}\|\vec{W}\|_{L^{\infty}} C_{R}+\|f\|_{L^{\infty}} C_{V}+C C_{\mu}\|f\|_{W_{\infty}^{1}}\right) .
\end{aligned}
$$

This proves first inequality from (15). The second inequality from (15) can be proved using (13) similarly. Thus, Proposition 1 is completely proved.

Proposition 2 Under the same assumptions for $\vec{W}$ and $V$ as in Proposition 1 there is a constant $k_{0}>1$ such that the function $u_{s c}(x, k, \theta)$ defined by the series

$$
u_{s c}(x, k, \theta)=\sum_{j=0}^{\infty} L_{k}^{j}\left(\tilde{u}_{0}\right)(x, k, \theta)
$$

solves integral equation (8) ((6)) uniquely in $W_{\infty}^{1}\left(R^{3}\right)$, when $k \geq k_{0}$. Moreover,

$$
\left\|u_{s c}\right\|_{L^{\infty}\left(R^{3}\right)} \leq \frac{2 c_{0}}{k}, \quad\left\|\nabla u_{s c}\right\|_{L^{\infty}\left(R^{3}\right)} \leq 2 c_{0}
$$

uniformly in $\theta \in S^{2}$, when $k \geq k_{0}$.

Proof The estimates (15) imply that the norm estimate for operator $L_{k}$ for $k \geq 1$ is

$$
\left\|L_{k}\right\|_{W_{\infty}^{1} \rightarrow W_{\infty}^{1}} \leq \frac{2 c_{0}}{k} .
$$

Since $\tilde{u}_{0}$ belongs to $W_{\infty}^{1}\left(R^{3}\right)$ this estimate in turn implies that

$$
\left\|u_{s c}\right\|_{W_{\infty}^{1}} \leq \sum_{j=0}^{\infty}\left(\frac{2 c_{0}}{k}\right)^{j}\left\|\tilde{u}_{0}\right\|_{W_{\infty}^{1}} .
$$


We may choose any $k_{0}>\max \left\{1,2 c_{0}\right\}$ to conclude that the series (16) converges in $W_{\infty}^{1}\left(R^{3}\right)$. Because the operator $L_{k}$ is linear and maps continuously in $W_{\infty}^{1}\left(R^{3}\right)$ the series (16) solves (8). Choosing now $k_{0}>\max \left\{1,4 c_{0}\right\}$ one can easily obtain (17). Uniqueness of solution follows from the contraction condition of $L_{k}$. Proposition 2 is completely proved.

Concerning the kernel $G_{p}$ (see integral equation (11)) of the integral operator $\left(H_{4}-k^{4}-i 0\right)^{-1}$ one can prove the following result.

Proposition 3 Under the same assumptions for $\vec{W}$ and $V$ as in Proposition 1 there is a constant $k_{0}>1$ such that the function $G_{p}(x, y, k)$ can be defined by the series

$$
\begin{gathered}
G_{p}(x, y, k)=\sum_{j=0}^{\infty} G^{(j)}(x, y, k), \quad G^{(0)}=G_{k}^{+}, \\
G^{(j)}(x, y, k):=-\int_{R^{3}} G_{k}^{+}(|x-z|)\left(\vec{W}(z) \cdot \nabla G^{(j-1)}(z, y, k)+V(z) G^{(j-1)}(y, z, k)\right) d z
\end{gathered}
$$

which solves integral equation (11) uniquely, when $k \geq k_{0}$. Moreover,

$$
\begin{gathered}
\left|G_{p}(x, y, k)-G_{k}^{+}(x, y, k)\right| \leq \frac{\tilde{c}_{0}}{4 \pi^{2} k^{3}|x-y|}, \\
\left|\nabla G_{p}(x, y, k)-\nabla G_{k}^{+}(x, y, k)\right| \leq \frac{\tilde{c}_{0}}{2 \pi^{2} k^{2}|x-y|},
\end{gathered}
$$

where $\tilde{c}_{0}=2\|\vec{W}\|_{L^{\infty}} C_{R}+C_{V}+3 C C_{\mu}$ with constants $C_{R}, C_{V}, C$ and $C_{\mu}$ are as in (14).

Proof To prove (18)-(19) it is needed first to estimate $G^{(1)}$. Indeed, using (13) one can obtain $(k \geq 1)$

$$
\left|G^{(1)}(x, y, k)\right| \leq \frac{1}{8 \pi^{2} k^{3}}\left(\int_{|z| \leq R} \frac{|\vec{W}(z)|+\frac{1}{2 k}|V(z)|}{|x-z||z-y|} d z+\int_{|z| \geq R} \frac{C+\frac{C}{2 k}}{|x-z||z-y||z|^{\mu}} d z\right)
$$

with constant $C$ from the condition (3). Considering now two cases: $|x-z| \leq$ $|z-y|$ and $|x-z| \geq|z-y|$ and taking into account conditions (2), one can obtain $(k \geq 1)$

$$
\begin{aligned}
\left|G^{(1)}(x, y, k)\right| \leq \frac{1}{8 \pi^{2} k^{3}} & \left(\frac{2\|\vec{W}\|_{L^{\infty}} C_{R}}{|x-y|}+\frac{C_{V}}{|x-y|}+\frac{3 C C_{\mu}}{|x-y|}\right) \leq \\
\leq & \frac{\tilde{c}_{0}}{8 \pi^{2} k^{3}|x-y|},
\end{aligned}
$$


where the constant $\tilde{c}_{0}$ is as above. Similarly one can obtain

$$
\left|\nabla_{x} G^{(1)}(x, y, k)\right| \leq \frac{\tilde{c}_{0}}{4 \pi^{2} k^{2}|x-y|} .
$$

We show now that for $k \geq 1$

$$
\left|G^{(j)}(x, y, k)\right| \leq \frac{\tilde{c}_{0}^{j}}{2 k(2 \pi k)^{j+1}} \frac{1}{|x-y|}, \quad j=1,2, \ldots,
$$

and

$$
\left|\nabla_{x} G^{(j)}(x, y, k)\right| \leq \frac{\tilde{c}_{0}^{j}}{(2 \pi k)^{j+1}} \frac{1}{|x-y|}, \quad j=1,2, \ldots .
$$

By (20) and (21) the claim holds for $j=1$. Suppose that the claim is proved for $j \geq 1$. The induction hypothesis leads to

$$
\begin{gathered}
\left|G^{(j+1)}(x, y, k)\right| \leq \frac{\tilde{c}_{0}^{j}}{4 \pi k^{2}(2 \pi k)^{j+1}}\left(\int_{z z \mid \leq R} \frac{|\vec{W}(z)|+\frac{1}{2 k}|V(z)|}{|x-z|} \frac{1}{|z-y|} d z+\right. \\
\left.+\int_{|z| \geq R} \frac{C+\frac{C}{2 k}}{|x-z||z|^{\mu}} \frac{1}{|z-y|} d z\right) \leq \frac{\tilde{c}_{0}^{j}}{4 \pi k^{2}(2 \pi k)^{j+1}}\left(\frac{2\|\mid \vec{W}\|_{L^{\infty} C_{R}}}{|x-y|}+\right. \\
\left.+\frac{C_{V}}{|x-y|}+\frac{3 C C_{\mu}}{|x-y|}\right)=\frac{\tilde{c}_{0}^{j}}{4 \pi k^{2}(2 \pi k)^{j+1}} \frac{\tilde{c}_{0}}{|x-y|} .
\end{gathered}
$$

This finishes the proof of (22) by induction. The estimate (23) can be obtained similarly (by induction). Choosing now $k_{0}>\max \left\{1, \frac{\tilde{c}_{0}}{\pi}\right\}$ we obtain the estimates (19). Thus, Proposition 3 is proved.

Next we study the asymptotic behaviour of the scattering solutions $u$ that provides the scattering data fo the inverse scattering problems in the foregoing section.

Proposition 4 Assume that $\vec{W}$ is bounded, $V$ belongs to the Kato space $K_{3}$ and both satisfy condition (3). Then for fixed $k \geq k_{0}$ the solution $u(x, k, \theta)$ to (6) ((8)) admits the representation

$$
u(x, k, \theta)=e^{i k x \cdot \theta}-\frac{1}{8 \pi} \frac{e^{i k|x|}}{k^{2}|x|} A\left(k, \theta^{\prime}, \theta\right)+o\left(\frac{1}{|x|}\right), \quad|x| \rightarrow \infty,
$$

The function $A\left(k, \theta, \theta^{\prime}\right)$ is called the scattering amplitude and is defined by equation (9).

\section{Proof Since}

$$
u_{s c}(y, k, \theta)=-\int_{R^{3}} G_{k}^{+}(|x-y|)(\vec{W}(y) \cdot \nabla u(y)+V(y) u(y)) d y=
$$




$$
\begin{gathered}
=-\int_{|y| \leq|x|^{a}} G_{k}^{+}(|x-y|)(\vec{W}(y) \cdot \nabla u(y)+V(y) u(y)) d y- \\
-\int_{|y| \geq|x|^{a}} G_{k}^{+}(|x-y|)(\vec{W}(y) \cdot \nabla u(y)+V(y) u(y)) d y=: I_{1}+I_{2},
\end{gathered}
$$

where parameter $a$ is chosen such that $0<a<\frac{1}{2}$. For the integral $I_{1}$ we use the following asymptotic

$$
G_{k}^{+}(|x-y|)=\frac{1}{8 \pi k^{2}}\left(e^{i k|x|} e^{-i k \theta^{\prime} \cdot y}-e^{-k|x|} e^{k \theta^{\prime} \cdot y}\right)+O\left(|x|^{2 a-2}\right) .
$$

This implies that

$$
I_{1}=-\frac{1}{8 \pi k^{2}} \int_{|y| \leq|x|^{a}}\left(e^{i k|x|} e^{-i k \theta^{\prime} \cdot y}-e^{-k|x|} e^{k \theta^{\prime} \cdot y}\right)(\vec{W} \cdot \nabla u+V u) d y+O\left(|x|^{2 a-2}\right) .
$$

And this in turn leads as $|x| \rightarrow+\infty$ to

$$
I_{1}=-\frac{e^{i k|x|}}{8 \pi k^{2}} \int_{R^{3}} e^{-i k \theta^{\prime} \cdot y}(\vec{W} \cdot \nabla u+V u) d y+o\left(|x|^{-1}\right) .
$$

Next we consider the integral $I_{2}$ and split the region of integration as

$$
\begin{gathered}
\left|I_{2}\right| \leq \int_{|y| \geq|x|^{a}}\left|G_{k}^{+}(|x-y|)\right||\vec{W}(y) \cdot \nabla u(y)+V(y) u(y)| d y= \\
=\int_{|x|^{a} \leq|y| \leq \frac{|x|}{2}}\left|G_{k}^{+}(|x-y|)\right||\vec{W}(y) \cdot \nabla u(y)+V(y) u(y)| d y+ \\
+\int_{|y| \geq \frac{|x|}{2}}\left|G_{k}^{+}(|x-y|)\right||\vec{W}(y) \cdot \nabla u(y)+V(y) u(y)| d y=: J_{1}+J_{2} .
\end{gathered}
$$

In the case $J_{1}$ we have $|x-y| \geq|x|-|y| \geq \frac{|x|}{2}$. Thus, as $|x| \rightarrow+\infty$, one can have

$$
J_{1} \leq \frac{1}{4 \pi k^{2}|x|} \int_{|x|^{a} \leq|y| \leq \frac{|x|}{2}}|\vec{W}(y) \cdot \nabla u(y)+V(y) u(y)| d y=o\left(|x|^{-1}\right)
$$

due to conditions (2), (3) and Proposition 1. To estimate the integral $J_{2}$ we use condition (3) and Proposition 1 and obtain

$$
J_{2} \leq \frac{c}{k^{2}} \int_{|y| \geq \frac{|x|}{2}} \frac{1}{|x-y||y|^{\mu}} d y \leq \frac{c}{k^{2}|x|^{\epsilon}} \int_{|y| \geq \frac{|x|}{2}} \frac{1}{|x-y||y|^{\mu-\epsilon}} d y,
$$


where positive $\epsilon$ is chosen such that $2<\mu-\epsilon<3$ and $c>0$. Apply now the estimate for the convolution of weak singularities (see, for example, [12], Lemma 34.3) one can finally obtain that

$$
J_{2} \leq \frac{c}{k^{2}|x|^{\mu-2}}=o\left(|x|^{-1}\right)
$$

since $\mu>3$. The latter estimates and (24) finish the proof of Proposition 4 .

\section{Proof of the main results}

This Section is devoted to the proof of the main results and their consequences.

Proof We prove Theorem 1. Indeed, denoting by $I$ the integral

$$
I:=k^{2} \int_{S^{2} \times S^{2}} e^{-i k\left(\theta-\theta^{\prime}\right) \cdot x} A\left(k, \theta, \theta^{\prime}\right) d \theta d \theta^{\prime}
$$

one can write (because $u=u_{0}+u_{s c}$ )

$$
\begin{gathered}
I:=k^{2} \int_{S^{2} \times S^{2}} e^{-i k\left(\theta-\theta^{\prime}\right) \cdot x} \int_{R^{3}} e^{-i k \theta^{\prime} \cdot y}[i k \theta \cdot \vec{W}(y)+V(y)] d y d \theta d \theta^{\prime}+ \\
+k^{2} \int_{S^{2} \times S^{2}} e^{-i k\left(\theta-\theta^{\prime}\right) \cdot x} \int_{R^{3}} e^{-i k \theta^{\prime} \cdot y}\left[\vec{W}(y) \cdot \nabla u_{s c}(y)+V(y) u_{s c}(y)\right] d y d \theta d \theta^{\prime}= \\
=: I_{1}+I_{2} .
\end{gathered}
$$

Since $\vec{W}$ and $V$ belong to $L^{1}\left(R^{3}\right)$ (see conditions (2) and (3)) then $I_{1}$ can be rewritten as

$$
\begin{aligned}
I_{1}= & k^{2} \int_{R^{3}} \vec{W}(y) d y \cdot \int_{S^{2}} i k \theta e^{-i k \theta \cdot(x-y)} d \theta \int_{S^{2}} e^{i k \theta^{\prime} \cdot(x-y)} d \theta^{\prime}+ \\
& +k^{2} \int_{R^{3}} V(y) d y \int_{S^{2}} e^{-i k \theta \cdot(x-y)} d \theta \int_{S^{2}} e^{i k \theta^{\prime} \cdot(x-y)} d \theta^{\prime} .
\end{aligned}
$$

It is very well known that (see, for example, [7])

$$
\int_{S^{2}} e^{-i k \theta \cdot(x-y)} d \theta=\sqrt{(2 \pi)^{3}} \frac{J_{\frac{1}{2}}(k|x-y|)}{\sqrt{k|x-y|}}=4 \pi \frac{\sin (k|x-y|)}{k|x-y|} .
$$

Hence, $I_{1}$ is equal to

$$
I_{1}=8 \pi^{2} \int_{R^{3}} \vec{W}(y) \cdot \nabla_{x}\left(\frac{\sin ^{2}(k|x-y|)}{|x-y|^{2}}\right) d y+16 \pi^{2} \int_{R^{3}} V(y) \frac{\sin ^{2}(k|x-y|)}{|x-y|^{2}} d y .
$$


If now $\varphi \in C_{0}^{\infty}\left(R^{3}\right)$ then in the sense of distributions

$$
\begin{aligned}
<I_{1}, \varphi> & =8 \pi^{2} \int_{R^{3}} \int_{R^{3}} \vec{W}(y) \cdot \nabla_{x}\left(\frac{\sin ^{2}(k|x-y|)}{|x-y|^{2}}\right) \varphi(x) d y d x+ \\
& +16 \pi^{2} \int_{R^{3}} \int_{R^{3}} V(y) \frac{\sin ^{2}(k|x-y|)}{|x-y|^{2}} \varphi(x) d y d x .
\end{aligned}
$$

Using the smoothness of $\varphi$ and compactness of its support and integrating by parts we obtain

$$
\begin{aligned}
&<I_{1}, \varphi>=-8 \pi^{2} \int_{R^{3}} \vec{W}(y) d y \int_{R^{3}} \frac{\sin ^{2}(k|x-y|)}{|x-y|^{2}} \nabla_{x} \varphi(x) d x+ \\
&+ 16 \pi^{2} \int_{R^{3}} V(y) d y \int_{R^{3}} \frac{\sin ^{2}(k|x-y|)}{|x-y|^{2}} \varphi(x) d x= \\
&=-4 \pi^{2} \int_{R^{3}} \vec{W}(y) d y \int_{R^{3}} \frac{1}{|x-y|^{2}} \nabla_{x} \varphi(x) d x+ \\
&+ 4 \pi^{2} \int_{R^{3}} \vec{W}(y) d y \int_{R^{3}} \frac{\cos (2 k|x-y|)}{|x-y|^{2}} \nabla_{x} \varphi(x) d x+ \\
&+8 \pi^{2} \int_{R^{3}} V(y) d y \int_{R^{3}} \frac{1}{|x-y|^{2}} \varphi(x) d x- \\
&-8 \pi^{2} \int_{R^{3}} V(y) d y \int_{R^{3}} \frac{\cos (2 k|x-y|)}{|x-y|^{2}} \varphi(x) d x .
\end{aligned}
$$

Then application of Fubini theorem and Riemann - Lebesgue lemma lead to the equality

$$
\lim _{k \rightarrow+\infty}<I_{1}, \varphi>=4 \pi^{2}<\nabla_{x} \int_{R^{3}} \frac{\vec{W}(y)}{|x-y|^{2}} d y, \varphi>+8 \pi^{2}<\int_{R^{3}} \frac{V(y)}{|x-y|^{2}} d y, \varphi>.
$$

To estimate $I_{2}$ one can first rewrite it (using again (25)) as

$$
\begin{aligned}
I_{2}= & 4 \pi k \int_{R^{3}} \frac{\sin (k|x-y|)}{|x-y|} \vec{W}(y) d y \cdot \int_{S^{2}} e^{-i k \theta \cdot x} \nabla u_{s c}(k, y, \theta) d \theta+ \\
& +4 \pi k \int_{R^{3}} \frac{\sin (k|x-y|)}{|x-y|} V(y) d y \int_{S^{2}} e^{-i k \theta \cdot x} u_{s c}(k, y, \theta) d \theta .
\end{aligned}
$$


Next, we use the following equalities (see, for example, [15] or [12])

$$
\begin{gathered}
u_{s c}(y)=-\hat{G}_{p}\left(\vec{W}(z) \cdot \nabla u_{0}(z)+V(z) u_{0}(z)\right)(y), \\
\nabla_{y} u_{s c}(y)=-\nabla_{y} \hat{G}_{p}\left(\vec{W}(z) \cdot \nabla u_{0}(z)+V(z) u_{0}(z)\right)(y),
\end{gathered}
$$

where $\hat{G}_{p}$ denotes the integral operator with kernel $G_{p}(x, y, k)$ (see Proposition 3 ). These equalities and (25) allow to obtain for $I_{2}$ the following representation

$$
\begin{aligned}
& -16 \pi^{2} \int_{R^{3}} \frac{\sin (k|x-y|)}{|x-y|} \vec{W}(y) \cdot \nabla_{y} \hat{G}_{p}\left(\frac{\sin (k|x-z|)}{|x-z|} V(z)+\vec{W}(z) \nabla_{x} \frac{\sin (k|x-z|)}{|x-z|}\right) d y- \\
& -16 \pi^{2} \int_{R^{3}} \frac{\sin (k|x-y|)}{|x-y|} V(y) \hat{G}_{p}\left(\frac{\sin (k|x-z|)}{|x-z|} V(z)+\vec{W}(z) \nabla_{x} \frac{\sin (k|x-z|)}{|x-z|}\right) d y .
\end{aligned}
$$

The estimates (13) and (19) for $G_{p}(x, y, k)$ and the same technique as for $I_{1}$ allow easily to obtain that for any $\varphi \in C_{0}^{\infty}\left(R^{3}\right)$

$$
\lim _{k \rightarrow+\infty}<I_{2}, \phi>=0 \text {. }
$$

This finishes the proof of Theorem 1.

Remark If we assume in addition that $\vec{W} \in W_{p}^{1}\left(R^{3}\right)$ and $V \in L^{p}\left(R^{3}\right)$ with some $3<p \leq \infty$ and with the same behaviour at the infinity then it can be proved that the limit in Saito's formula holds uniformly in $x \in R^{3}$ (see [15], [9]).

Proof We prove Corollary 1 (Uniqueness). We have only to show that the homogeneous equation

$$
\Psi(x):=\frac{1}{2} \nabla_{x} \int_{R^{3}} \frac{\vec{W}(y)}{|x-y|^{2}} d y+\int_{R^{3}} \frac{V(y)}{|x-y|^{2}} d y=0
$$

has a unique solution such that $\frac{1}{2} \nabla \delta \star \vec{W}-V=0$. Indeed, following [11] and [10] consider the space $S_{0}\left(R^{3}\right)$ of all functions from the Schwarz space which vanish in some neighborhood of the origin. Then for every $\varphi(\xi) \in S_{0}\left(R^{3}\right)$ it follows that

$$
\begin{gathered}
0=<\mathcal{F}(\Psi(x))(\xi), \varphi(\xi)>=2 \pi^{2}<-\frac{i \xi}{2|\xi|} \cdot \mathcal{F}(\vec{W})(\xi)+\frac{\mathcal{F}(V)(\xi)}{|\xi|}, \varphi(\xi)>= \\
=2 \pi^{2}<-\frac{i \xi}{2} \cdot \mathcal{F}(\vec{W})(\xi)+\mathcal{F}(V)(\xi), \frac{\varphi(\xi)}{|\xi|}>,
\end{gathered}
$$

where $\mathcal{F}$ is usual Fourier transform in $R^{3}$. Since $\varphi(\xi) \in S_{0}\left(R^{3}\right)$ then $\frac{\varphi(\xi)}{|\xi|} \in$ $S_{0}\left(R^{3}\right)$ also. Hence, for every $h \in S_{0}\left(R^{3}\right)$, one can see that

$$
<\frac{i \xi}{2} \cdot \mathcal{F}(\vec{W})(\xi)-\mathcal{F}(V)(\xi), h(\xi)>=0 .
$$


This means that the support of the function $\frac{i \xi}{2} \cdot \mathcal{F}(\vec{W})(\xi)-\mathcal{F}(V)(\xi)$ is at most in the origin, and therefore it can be represented as follows (with some integer $m$ )

$$
\frac{i \xi}{2} \cdot \mathcal{F}(\vec{W})(\xi)-\mathcal{F}(V)(\xi)=\sum_{|\alpha| \leq m} C_{\alpha} \partial^{\alpha} \delta(\xi),
$$

where $\delta(\xi)$ is Dirac $\delta$-function and $C_{\alpha}$ are constants. The conditions for $\vec{W}$ and $V$ imply that all these constants are equal to 0 . Thus,

$$
\mathcal{F}^{-1}\left(\frac{i \xi}{2} \cdot \mathcal{F}(\vec{W})(\xi)-\mathcal{F}(V)(\xi)\right)=0, \quad \text { i.e. } \quad \frac{1}{2} \nabla \delta \star \vec{W}(x)-V(x)=0 .
$$

This finishes the proof of Corollary 1.

Proof We prove now Theorem 2 (Reconstruction). Based on Proposition 3, one can represent (see (11) and (18))

$$
\begin{gathered}
G_{k}^{+}(|x-y|)-G_{p}(x, y, k)=\int_{R^{3}} G_{k}^{+}(|x-z|)\left[\vec{W}(z) \cdot \nabla_{z} G_{p}(z, y, k)+V(z) G_{p}(z, y, k)\right] d z= \\
=\int_{R^{3}} G_{k}^{+}(|x-z|)\left[\vec{W}(z) \cdot \nabla_{z} G_{k}^{+}(|z-y|)+V(z) G_{k}^{+}(|z-y|)\right] d z+ \\
+\int_{R^{3}} G_{k}^{+}(|x-z|)\left[\vec{W}(z) \cdot \nabla_{z} \sum_{j=1}^{\infty} G^{(j)}(z, y, k)+V(z) \sum_{j=1}^{\infty} G^{(j)}(z, y, k)\right] d z:=J_{1}+J_{2},
\end{gathered}
$$

where $k \geq k_{0}$. We first consider $J_{1}$ and divide it into to parts $J_{1}^{\prime}$ and $J_{1}^{\prime \prime}$ w.r.t. $|z|<k$ and $|z|>k$, respectively for fixed $k$ big enough. Using conditions (3) and estimates (13) the value $J_{1}^{\prime \prime}$ can be estimated as

$$
\left|J_{1}^{\prime \prime}\right| \leq \frac{c}{k^{3}} \int_{|z|>k} \frac{1}{|x-z||z-y||z|^{\mu}} d z
$$

where constant $c>0$ is independent on $x, y, k$. Since $\mu>4$ then the latter integral is $o\left(\frac{1}{k^{4}|x||y|}\right)$ uniformly in $k, x, y$ big enough. For estimation of $J_{1}^{\prime}$ we assume that $|x|>k^{4+s},|y|>k^{4+s}$ with $s>0$ and with $k$ is big enough. In this case

$$
i k(z-y)=-|y| i k \theta^{\prime}+i k z, \quad i k|x-z|=i k|x|-i k \theta \cdot z+O\left(k^{-1-s}\right),
$$

where $\theta=\frac{x}{|x|}, \theta^{\prime}=\frac{y}{|y|}$. These representations lead to the following asymptotic behaviour

$$
G_{k}^{+}(|x-z|)=\frac{e^{i k|x|} e^{-i k \theta \cdot z}}{8 \pi k^{2}|x|}\left(1+\frac{O(1)}{k^{1+s}}\right), \quad|z|<k
$$




$$
\nabla_{z} G_{k}^{+}(|y-z|)=\frac{e^{i k|y|} e^{-i k \theta^{\prime} \cdot z}}{8 \pi k^{2}|y|}\left(1+\frac{O(1)}{k^{1+s}}\right)\left(-i k \theta^{\prime}+\frac{i k z}{|y|}\right), \quad|z|<k .
$$

This allows to obtain that

$$
J_{1}^{\prime}=-\frac{e^{i k(|x|+|y|)}}{64 \pi^{2} k^{4}|x||y|} \int_{|z|<k}\left(i k \vec{W}(z) \cdot \theta^{\prime}-V(z)\right) e^{-i k\left(\theta+\theta^{\prime}\right) \cdot z} d z+O\left(\frac{1}{k^{4+s}|x||y|}\right) .
$$

The next step is: symmetric (w.r.t. $\theta$ and $\theta^{\prime}$ ) form of the latter integral

$$
\int_{|z|<k} i k \vec{W}(z) \cdot \theta^{\prime} e^{-i k\left(\theta+\theta^{\prime}\right) \cdot z} d z
$$

leads to the fact that

$$
\int_{|z|<k} i k \vec{W}(z) \cdot \theta^{\prime} e^{-i k\left(\theta+\theta^{\prime}\right) \cdot z} d z=\frac{1}{2} \int_{|z|<k} i k \vec{W}(z) \cdot\left(\theta^{\prime}+\theta^{\prime}\right) e^{-i k\left(\theta+\theta^{\prime}\right) \cdot z} d z .
$$

Hence, the first term of the sum (27) can be represented as

$$
-\frac{e^{i k(|x|+|y|)}}{64 \pi^{2} k^{4}|x||y|} \int_{|z|<k}\left(\frac{i k}{2} \vec{W}(z) \cdot\left(\theta+\theta^{\prime}\right)-V(z)\right) e^{-i k\left(\theta+\theta^{\prime}\right) \cdot z} d z .
$$

For estimation of the term $J_{2}$ one can use the estimates (19), (22), (23) and conditions (2) and (3). Then using quite similar technique (with more careful considerations of $G^{(2)}$ ) as for the proof of the estimates (27) and (28) one can obtain that

$$
J_{2}=o\left(\frac{1}{k^{4}|x||y|}\right), \quad k,|x|,|y| \rightarrow+\infty .
$$

Letting now $k,|x|,|y| \rightarrow+\infty$ such that fixed $\xi=k\left(\frac{x}{|x|}+\frac{y}{|y|}\right)$ and combining now (26) - (29) we obtain formula (12). Thus, Theorem 2 is completely proved.

Corollary 2 can be proved similarly as the proof of Corollary 1 .

\section{Conclusions}

The direct scattering problems for a first order perturbation of the threedimensional biharmonic operator with singular coefficients $\left(\vec{W}\right.$ belongs to $L_{l o c}^{\infty}$ and $V$ belongs to the Kato space $K_{3, l o c}$ ) was studied. It was shown that a solution to scattering problem with certain radiation conditions satisfies the integral Lippmann-Schwinger equation. The same was shown for the Green's function of the perturbed biharmonic operator with these coefficients. These integral equations have the unique solutions in the usual Sobolev spaces $W_{\infty}^{1}\left(R^{3}\right)$. The asymptotic behaviour of the scattering solutions for fixed $k>0$ as $|x| \rightarrow+\infty$ 
was studied and a formula for the scattering amplitude was obtained. Similar asymptotic is obtained for the Green's function when $k,|x|,|y| \rightarrow+\infty$.

The main results of this paper, Saito's formula and formula for the Green's functions of the bi-Laplacian and the perturbed bi-Laplacian, were proved under very general assumptions on the coefficients. The proof of these formulas itself was based on explicit calculations starting from the formula for scattering amplitude and from the integral equation for the Green's functions. Some consequences of these results were discussed w.r.t. inverse scattering problems. Namely, the scattering amplitude uniquely determines a combination of the coefficients for the direct problem and in turn gives a uniqueness result for the inverse problem. And the behaviour of the Green's function of the perturbed bi-Laplacian for $x, y, k$ large enough uniquely determines the same combination of the unknown coefficients.

\section{Acknowledgments}

This work was supported by the Academy of Finland (application no 312123, Finnish Programme for Centres of Excellence in Inverse Modelling and Imaging 2018-2025).

\section{References}

[1] Aktosun, T. and Papanicolaou, V.G. (2008), Time-evolution of the scattering data for a fourth-order linear differential operator, Inverse Problems, Vol. 24, 055013, $14 \mathrm{pp}$.

[2] Gazola, F., Grumau, H.-C., and Sweers, G., Polyharmonic Boundary Value Problems, Springer - Verlag, Berlin, Heidelberg, 2010.

[3] Harju, M., On the direct and inverse scattering problems for a nonlinear three-dimensional Schrödinger equation, Doctoral dissertation, University of Oulu, 2010.

[4] Iwasaki, K., Scattering theory for the 4th order differential operators: I (1988), Japan. J. Math., Vol. 14, pp. 1-57.

[5] Iwasaki, K., Scattering theory for the 4th order differential operators: II (1988), Japan. J. Math., Vol. 14, pp. 59-96.

[6] Krupchyk, K., Lassas, M. and Uhlmann, G. (2014), Inverse boundary value problems for the perturbed poly-harmonic operator, Trans. Amer. Math. Soc., Vol. 36, pp. 95-112.

[7] Lebedev, N.N., Special Functions and Their Applications, Dover Publications, New York, 1972. 
[8] Pausader, B., Scattering for the defocusing beam equation in low dimensions (2010), Indiana Univ. Math. J., Vol. 58, pp. 791-822.

[9] Päivärinta, L. and Serov, V. (1998), Recovery of singularities of a multidimensional scattering potential, SIAM J. Math. Anal., Vol. 29, pp. 697-711.

[10] Razborov, A.G., Sagyndykov, M.K. and Serov, V.S. (2002), Some inverse problems for the Schrödinger operator with Kato potential, Inverse Ill-posed Problems, Vol. 10, pp. 395-411.

[11] Saito, Y. (1982), Some properties of the scattering amplitude and the inverse scattering problem, Osaka J. Math. Vol. 19, pp. 527-547.

[12] Serov, V., Fourier Series, Fourier Transform and Their Applications to Mathematical Physics, Springer, New-York, 2017.

[13] Sylvester, J. and Uhlmann, G. (1987), A global uniqueness theorem for an inverse boundary value problem, Ann. Math., Vol. 125, pp. 153-169.

[14] Sun, Z. (1993), An inverse boundary value problem for Schrödinger operators with vector potentials, Trans. AMS, Vol. 338, pp. 953-969.

[15] Tyni, T. and Serov, V. (2018), Scattering problems for perturbations of the multidimensional biharmonic operator, Inverse Problems and Imaging, Vol. 12(1), pp. 205-227. 